\title{
Burdens of Invasive Methicillin-Susceptible and Methicillin-Resistant Staphylococcus aureus Disease, Minnesota, USA
}

\section{Mackenzie Koeck, Kathryn Como-Sabetti, Dave Boxrud, Ginette Dobbins, Anita Glennen, Melissa Anacker, Selina Jawahir, Isaac See, Ruth Lynfield}

During August 1, 2014-July 31, 2015, in 2 counties in Minnesota, USA, incidence of invasive methicillin-susceptible Staphylococcus aureus (MSSA) (27.1 cases/100,000 persons) was twice that of invasive methicillin-resistant $S$. aureus (13.1 cases/100,000 persons). MSSA isolates were more genetically diverse and susceptible to more antimicrobial drugs than methicillin-resistant $S$. aureus isolates.

$\mathrm{M}$ ethicillin-resistant Staphylococcus aureus (MRSA) infections were first reported in the 1960s in healthcare facilities (1). Risk factors included recent hospitalization, surgery, dialysis, having a central venous catheter or other invasive medical device, chronic wounds, residence in long-term care facilities or prisons, injection drug use, and exposure to antimicrobial drugs (2). In the 1990s, MRSA infections caused by genetically distinct strains were observed among healthy persons in the community (3).

Although $S$. aureus has long been recognized as a major human pathogen, epidemiologic studies and infection prevention precautions in recent decades have largely focused on MRSA. We compared epidemiologic, microbiologic, and molecular characteristics of invasive methicillin-susceptible $S$. aureus (MSSA) infections with those of invasive MRSA infections.

\section{The Study}

Active surveillance for invasive MSSA was added to existing invasive MRSA surveillance in Ramsey and Hennepin Counties (combined population in 2014: 1,742,806) in Minnesota, USA, during 2014. Cases were defined as $S$. aureus isolated from a normally sterile site in a catchment

Author affiliations: Minnesota Department of Health, St. Paul, Minnesota, USA (M. Koeck, K. Como-Sabetti, D. Boxrud,

G. Dobbins, A. Glennen, M. Anacker, S. Jawahir, R. Lynfield); Centers for Disease Control and Prevention, Atlanta, Georgia, USA (I. See)

DOI: https://doi.org/10.3201/eid2501.181146 area resident. We collected demographic and clinical information from medical record reviews by using a standardized case report form. We epidemiologically characterized patients with invasive $S$. aureus: hospital onset (culture obtained $>3$ days after admission); healthcare-associated, community onset (HACO; $\geq 1$ healthcare-associated risk factor); and community associated (CA). Healthcare-associated risk factors included in the year before culture hospital admission or residence in a long-term care facility or long-term acute-care facility for any duration, surgery, or dialysis or within 2 days before culture presence of a central vascular catheter.

Invasive MSSA and MRSA isolates were submitted to the Minnesota Department of Health for species confirmation, antimicrobial drug susceptibility testing, and molecular characterization. We confirmed species by using the tube coagulase test (Becton Dickinson, http://www. bd.com) or matrix-assisted laser desorption ionization time-of-flight mass spectrometry by using a Microflex LT/SH mass spectrometer and FlexControl 3.4 software (Bruker Daltonics, Inc., https://www.bruker.com). We subtyped isolates by using pulsed-field gel electrophoresis (PFGE) according to published protocols (4). PFGE patterns were analyzed by using BioNumerics software (Applied Maths, http://www.applied-maths.com); patterns without differences were considered indistinguishable. We assigned patterns a USA clonal group on the basis of an $80 \%$ similarity coefficient cutoff to a type strain. Whole-genome sequencing characterized 2 isolates that were untypable by PFGE. We determined sequence type by using short reads in Illumina Miseq as described (5) and retrieved sequences by uploading raw read files to the Multilocus Sequence Typing 1.8 server ( $S$. aureus configuration) hosted by the Center for Genomic Epidemiology (6).

We performed antimicrobial drug susceptibility testing by using broth microdilution with a custom dried panel (TREK Diagnostic Systems, Inc., http://www.trekds.com/ techInfoVT/default.asp) and interpreted results according to Clinical and Laboratory Standards Institute guidelines (7). Isolates susceptible to penicillin were examined for $\beta$-lactamase production by using nitrocefin disk test, penicillin zone-edge test, and blaZ $\beta$-lactamase gene confirmation by PCR. We induced $\beta$-lactamase production for 
nitrocefin disk testing by inoculating an isolate onto sheep blood agar with a $1-\mu \mathrm{g}$ oxacillin disk and incubating overnight by using TREK Diagnostic Systems. Subsequent growth from the zone periphery was incubated with a nitrocefin disk (REMEL, http://www.remel.com/About.aspx) for $60 \mathrm{~min}$. We also performed a penicillin zone-edge test by using standard disk diffusion, followed by assessment for fuzzy or sharp zone edges. We determined the incidence of the blaZ $\beta$-lactamase gene by using real-time PCR as described but with slight modifications (8).

We analyzed data by using SAS version 9.4 (SAS Institute, https://www.sas.com). Census data for 2014 were

\begin{tabular}{|c|c|c|c|c|c|c|c|c|c|c|c|c|}
\hline \multirow[t]{2}{*}{ Characteristic } & \multicolumn{3}{|c|}{ Total, $\mathrm{n}=701$} & \multicolumn{3}{|c|}{$\mathrm{HO}, \mathrm{n}=65$} & \multicolumn{3}{|c|}{ HACO, $n=416$} & \multicolumn{3}{|c|}{$\mathrm{CA}, \mathrm{n}=220$} \\
\hline & MSSA & MRSA & $\begin{array}{c}\mathrm{p} \\
\text { value }\end{array}$ & MSSA & MRSA & $\begin{array}{c}\mathrm{p} \\
\text { value }\end{array}$ & MSSA & MRSA & $\begin{array}{c}\mathrm{p} \\
\text { value }\end{array}$ & MSSA & MRSA & $\begin{array}{c}\mathrm{p} \\
\text { value }\end{array}$ \\
\hline Total & $\begin{array}{l}473 \\
(67)\end{array}$ & $\begin{array}{l}228 \\
(33) \\
\end{array}$ & NA & $40(8)$ & $25(11)$ & 0.28 & $\begin{array}{l}260 \\
(55)\end{array}$ & $\begin{array}{l}156 \\
(68)\end{array}$ & $<0.001$ & $173(37)$ & $47(21)$ & $<0.01$ \\
\hline \multicolumn{13}{|l|}{ Demographic } \\
\hline Median age, $y$ & $\begin{array}{c}59(0- \\
102)\end{array}$ & $\begin{array}{c}62(0- \\
95)\end{array}$ & 0.05 & $\begin{array}{c}61(0- \\
94)\end{array}$ & $\begin{array}{c}61(17- \\
93)\end{array}$ & 0.99 & $\begin{array}{c}62(0- \\
102)\end{array}$ & $\begin{array}{c}65(5- \\
95)\end{array}$ & 0.21 & $\begin{array}{c}55(0- \\
90)\end{array}$ & $\begin{array}{l}50(0- \\
91)\end{array}$ & 0.40 \\
\hline \multicolumn{13}{|l|}{ Sex } \\
\hline $\mathrm{F}$ & $\begin{array}{l}175 \\
(37)\end{array}$ & $92(40)$ & 0.39 & $15(38)$ & $6(24)$ & 0.26 & $\begin{array}{l}108 \\
(42)\end{array}$ & $66(42)$ & 0.88 & $52(30)$ & $20(43)$ & 0.11 \\
\hline M & $\begin{array}{l}316 \\
(67)\end{array}$ & $\begin{array}{l}153 \\
(67)\end{array}$ & 0.94 & $26(65)$ & $13(52)$ & 0.30 & $\begin{array}{l}169 \\
(65)\end{array}$ & $\begin{array}{l}109 \\
(70)\end{array}$ & 0.31 & $121(70)$ & $31(66)$ & 0.60 \\
\hline $\begin{array}{l}\text { Private residence } 4 \mathrm{~d} \\
\text { before culture }\end{array}$ & $\begin{array}{l}376 \\
(79)\end{array}$ & $\begin{array}{l}146 \\
(64)\end{array}$ & $<0.01$ & NA & NA & NA & $\begin{array}{l}211 \\
(81)\end{array}$ & $\begin{array}{l}100 \\
(64)\end{array}$ & $<0.01$ & $165(95)$ & $46(98)$ & 0.69 \\
\hline LTCF past year & $53(11)$ & $70(31)$ & $<0.01$ & $4(10)$ & $8(32)$ & 0.05 & $49(19)$ & $62(40)$ & $<0.01$ & NA & NA & NA \\
\hline CVC 2 d before & $29(6)$ & $19(8)$ & 0.28 & $6(15)$ & $5(20)$ & 0. & $23(9)$ & $14(9)$ & 0.97 & NA & NA & NA \\
\hline Length of stay, $\mathrm{d}$ & $\begin{array}{l}7(0- \\
121)\end{array}$ & $\begin{array}{c}8(0- \\
58)\end{array}$ & 0.05 & $\begin{array}{c}15(3- \\
121)\end{array}$ & $\begin{array}{c}15(4- \\
48)\end{array}$ & 0.91 & $\begin{array}{c}6(0- \\
43)\end{array}$ & $\begin{array}{c}7(1- \\
39)\end{array}$ & 0.11 & $7(0-53)$ & $\begin{array}{c}9(0- \\
58)\end{array}$ & 0.15 \\
\hline Inpatient death & $47(10)$ & $26(11)$ & 0.55 & $8(20)$ & $2(8)$ & 0. & $29(11)$ & $23(15)$ & 0.2 & $10(6)$ & $1(2)$ & 0.46 \\
\hline \multicolumn{13}{|l|}{ Concurrent condition } \\
\hline None & $96(20)$ & $27(12)$ & $<0.01$ & $1(4)$ & $5(13)$ & 0.39 & $14(9)$ & $35(12)$ & 0.17 & $12(26)$ & $56(12)$ & 0.37 \\
\hline Kidr & $95(20)$ & $67(29)$ & $<0.0$ & & 9 & 0. & $77(30)$ & 54 & 0. & 9 & $4(9)$ & 0.48 \\
\hline $\begin{array}{l}\text { Chron } \\
\text { oreakdov }\end{array}$ & $22(5)$ & 25 (11) & $<0.01$ & $<0.01$ & $3(1$ & 0.6 & $14(5)$ & $21(13)$ & $<0$ & 5 & $1(2)$ & 1.00 \\
\hline D/PU & $9(2)$ & $17(7)$ & $<0.01$ & (0) & $0(0)$ & NA & $6(2)$ & $17(11)$ & $<0.01$ & $3(2)$ & $0(0)$ & 1.00 \\
\hline CVD & $58(12)$ & $46(20)$ & $<0$. & 5 & 6 & & 44 (17) & 39 & & & & 0.69 \\
\hline & $15(3)$ & $23(10)$ & $<0$. & & & & $9(3)$ & 20 & & & & 1.00 \\
\hline g use $\dagger$ & 41 (9) & 25 (11) & 0.3 & $4(10)$ & 2( & 1. & $21(8)$ & $11(7)$ & 0 . & $16(9)$ & $12(26)$ & $<0.01$ \\
\hline PV & 24 (5) & 22 (10) & 0. & & & & & & & & & 1.00 \\
\hline COP & 50 (11) & 34 (15) & 0.1 & $6(15)$ & $6(24)$ & 0. & 30 (12) & $23(15)$ & 0. & $14(8)$ & $5(11)$ & 0.57 \\
\hline Curr & 71 (15) & $32(14)$ & 0.7 & & & 1. & 4) & 19 (12) & 0. & $28(16)$ & 9 (19) & 0.63 \\
\hline Diabete & $\begin{array}{l}153 \\
(32)\end{array}$ & $80(35)$ & 0.47 & $12(30)$ & $11(44)$ & 0.25 & $96(37)$ & $59(38)$ & 0.86 & $45(26)$ & $10(21)$ & 0.51 \\
\hline Inje & $25(5)$ & $12(5)$ & & & & & & & & & & 0.11 \\
\hline Ob & & & & & & & & 29 & & & $7(15)$ & 0. \\
\hline \multicolumn{13}{|l|}{ Syndrome } \\
\hline Bact & $\begin{array}{l}328 \\
(69)\end{array}$ & $\begin{array}{l}178 \\
(78)\end{array}$ & 0.03 & $30(75)$ & $16(64)$ & 0.34 & $\begin{array}{l}181 \\
(70)\end{array}$ & $\begin{array}{l}125 \\
(80)\end{array}$ & 0.03 & $117(68)$ & $37(79)$ & 0.14 \\
\hline Inter & $25(5)$ & $11(5)$ & 0.7 & $2(5$ & 0 & 0. & $16(6)$ & $5(3)$ & 0. & $7(4)$ & $6(13)$ & 0.04 \\
\hline & 39 (8) & 40 (18) & $<0$. & & & & $21(8)$ & 22 & & & $10(21)$ & 0.02 \\
\hline Septic & $\begin{array}{l}137 \\
(29)\end{array}$ & 35 (15) & $<0.01$ & $3(8)$ & $2(8)$ & 1.00 & $67(26)$ & $24(15)$ & 0.01 & 67 (39) & $9(19)$ & 0.01 \\
\hline Ski & $7(1)$ & 4( & & & & & & 0 & 0. & & & $<0.01$ \\
\hline & 35 (7) & $10(4)$ & & & & & 7 (3) & 3 & & & $6(13)$ & 0.57 \\
\hline & $73(15)$ & 32 (14) & 0.60 & $6(15)$ & $3(12)$ & 1. & 38 (15) & $18(11)$ & 0. & 29 (17) & $11(23)$ & 0.30 \\
\hline En & $23(5)$ & $9(4)$ & 0.5 & $2(5)$ & $1(4)$ & 1. & $12(5)$ & $5(3)$ & 0. & 9 (5) & $3(6)$ & 0.72 \\
\hline Osteor & 51 (11) & $28(12)$ & 0.5 & $1(3)$ & $4(16)$ & 0. & 38 (15) & 20 (13) & 0. & $12(7)$ & $3(7)$ & 0.75 \\
\hline Septic shock & $41(9)$ & $19(8)$ & 0.86 & $3(8)$ & $4(16)$ & 0.42 & 27 (10) & $11(7)$ & 0.24 & 11 (6) & $4(9)$ & 0.53 \\
\hline
\end{tabular}

*Values are no. (\%), median (range), or no. (range). Nonsignificant ( $p \geq 0.05)$ differences were observed for chronic pulmonary disease, current smoker, diabetes, intravenous drug use, obesity, bursitis, cellulitis, endocarditis, osteomyelitis, and septic shock among patients with MSSA and MRSA cases and within epidemiologic classifications of MSSA and MRSA cases. CA, community associated; COPD, chronic pulmonary disease; CVC, central venous catheter; CVD, cardiovascular disease; D/PU, decubitus/pressure ulcer; HACO, healthcare-associated, community onset; $\mathrm{HO}$, hospital onset; $\mathrm{H} / \mathrm{P}$, hemiplagia/paraplegia; LTCF, long-term care facility; MRSA, methicillin-resistant Staphylococcus aureus; MSSA, methicillin-susceptible S. aureus; NA, not available; PVD, peripheral vascular disease.

†Drug use other than intravenous drug use.

$\ddagger$ All patients with pneumonia had positive blood cultures to meet the case definition. 
used to calculate incidence. We used a Wald $\chi^{2}$ test and $\mathrm{p} \leq 0.05$ to distinguish statistical significance. Infections with invasive MSSA and MRSA are reportable conditions under Minnesota State Rules, and obtaining information for surveillance purposes does not require Institutional Review Board review.

A total of 701 cases (473 invasive MSSA, 228 invasive MRSA) were reported during August 1, 2014-July 31, 2015. Incidence for invasive MSSA (27.1 cases/100,000 population) was more than twice that for invasive MRSA (13.1 cases $/ 100,000$ population) $(\mathrm{p}<0.001)$. Invasive MSSA cases were less likely to be HACO $(\mathrm{p}<0.001)$, more likely to be CA $(p<0.001)$, and have no concurrent conditions $(\mathrm{p}=0.006)$ than invasive MRSA cases. Bacteremia $(\mathrm{p}=$ $0.026)$ and pneumonia $(p<0.001)$ were less common among invasive MSSA cases. However, septic arthritis $(\mathrm{p}<0.001)$ was more common. Injection drug use and inpatient casefatality rate were similar for persons with invasive MSSA (6\% and $10 \%$, respectively) and invasive MRSA (5\% and $11 \%$, respectively) (Table 1 ).

Isolates from $40 \%$ of MSSA cases and $52 \%$ of MRSA cases were submitted. Cases with and without submitted isolates did not differ by sex, race, median age, or inpatient case-fatality rate. However, case-patients without isolates were more likely to have invasive MSSA $(p=0.003)$ or to be hospitalized in the year before culture $(\mathrm{p}=0.030)$.
Invasive MSSA isolates were more genetically diverse than invasive MRSA isolates. We could not assign a USA clonal group for $55 \%$ of invasive MSSA isolates compared with $29 \%$ of invasive MRSA isolates. The most common clonal group among invasive MSSA isolates was USA200, which included $14 \%$ of invasive MSSA isolates; $35 \%$ of invasive MRSA isolates were clonal group USA100, and $30 \%$ were USA300 (Table 2). The 2 invasive MSSA isolates that were untypable by PFGE were characterized by whole-genome sequencing as sequence type 398 ; 1 was from a HACO case and 1 was from a hospital onset case.

Invasive MSSA isolates were more often susceptible than invasive MRSA isolates to all antimicrobial drug classes, except for tetracycline (Table 2). We detected penicillin susceptibility through multiple testing methods in 63 (33\%) of invasive MSSA isolates. Six clonal groups were identified among penicillin-susceptible isolates.

\section{Conclusions}

The incidence of invasive MSSA was more than twice that of invasive MRSA in these counties. Invasive $S$. aureus infections were associated with a high case-fatality rate. Infection types were similar except for more frequent septic arthritis among invasive MSSA cases and bacteremia and pneumonia among invasive MRSA cases; these findings were consistent with those of other studies (9). Some

\begin{tabular}{|c|c|c|c|}
\hline Characteristic & MSSA, no. $(\%), n=190$ & MRSA, no. $(\%), n=119$ & $p$ value \\
\hline \multicolumn{4}{|l|}{ Clonal group } \\
\hline USA 100 & $13(7)$ & $42(35)$ & $<0.01$ \\
\hline USA200 & $26(14)$ & $0(0)$ & $<0.01$ \\
\hline USA300 & $5(3)$ & $36(30)$ & $<0.01$ \\
\hline USA400 & $1(1)$ & $3(3)$ & 0.16 \\
\hline USA500 & $2(1)$ & $0(0)$ & 0.56 \\
\hline USA600 & $13(7)$ & $0(0)$ & $<0.01$ \\
\hline USA700 & $5(3)$ & $3(3)$ & 1.00 \\
\hline USA900 & $10(5)$ & $0(0)$ & $<0.01$ \\
\hline USA1000 & $6(3)$ & $0(0)$ & 0.09 \\
\hline USA1200 & $2(1)$ & $0(0)$ & 0.56 \\
\hline Untypeable & $2(1)$ & $0(0)$ & 0.56 \\
\hline No USA group & $105(55)$ & $35(29)$ & $<0.01$ \\
\hline \multicolumn{4}{|l|}{ Antimicrobial drug $†$} \\
\hline Clindamycin $\ddagger$ & $176(93)$ & $61(51)$ & $<0.01$ \\
\hline Ceftaroline & $190(100)$ & $117(98)$ & 0.15 \\
\hline Doxycycline & $185(97)$ & $118(99)$ & 0.41 \\
\hline Erythromycin & $143(75)$ & $18(15)$ & $<0.01$ \\
\hline Gentamicin & 189 (99) & $118(99)$ & 1.00 \\
\hline Levofloxacin & $175(92)$ & $35(29)$ & $<0.01$ \\
\hline Penicillin & $63(33)$ & $0(0)$ & $<0.01$ \\
\hline Rifampin & $189(99)$ & $118(99)$ & 1.00 \\
\hline Tetracycline & $177(93)$ & $118(99)$ & 0.01 \\
\hline Trimethoprim/sulfamethoxazole & $188(99)$ & $119(100)$ & 0.53 \\
\hline
\end{tabular}

*MRSA, methicillin-resistant Staphylococcus aureus; MSSA, methicillin-susceptible S. aureus.

†Proportions represent isolates susceptible to an antimicrobial drug. All isolates were susceptible to daptomycin, linezolid, telavancin, and vancomycin A total of invasive MSSA and MRSA isolates had vancomycin MICs $<0.05 ; 86 \%$ of invasive MSSA isolates and $81 \%$ of invasive MSSA isolates had vancomycin MICs $=1 ; 13 \%$ of invasive MSSA isolates and $18 \%$ of invasive MRSA isolates had vancomycin MICs $=2$.

†lsolates with inducible clindamycin resistance were classified as nonsusceptible. 
studies found a higher case-fatality rate for MRSA than MSSA infections, possibly attributable to the older age and concurrent conditions among invasive MRSA case-patients (10). Although case-patients with invasive MSSA had fewer concurrent conditions and were less likely to have pneumonia (a syndrome associated with poor outcomes [11]) than case-patients with invasive MRSA, case-fatality rates were similar.

Invasive MSSA isolates were susceptible to more antimicrobial drugs and were more genetically diverse than invasive MRSA isolates, consistent with results of other reports (12). Penicillin susceptibility was observed in $33 \%$ of invasive MSSA isolates, which is considerably higher than for previous studies of invasive and noninvasive MSSA isolates and was seen for multiple strain types $(13,14)$.

Infection control interventions have effectively decreased healthcare-associated invasive MRSA incidence (15). However, invasive $S$. aureus burden and mortality rates remain a concern. Most invasive $S$. aureus disease was HACO or CA, highlighting the need for preventing these community-onset infections through new approaches and infection prevention in settings outside acute care. Ongoing surveillance data can inform planning for future interventions, such as improved wound care, enhanced infection prevention in nursing homes and dialysis centers, and greater attention to chronic conditions and development of effective vaccines.

This study was supported in part by a cooperative agreement (U50/CCU511190) with the Centers for Disease Control and Prevention as part of the Emerging Infections Program.

\section{About the Author}

Ms. Koeck is an epidemiologist in the Emerging Infections Unit, Minnesota Department of Health, St. Paul, MN. Her primary research interest is coordinating surveillance for invasive S. aureus.

\section{References}

1. Jevons M. Celbenin-resistant staphylococci. BMJ. 1961;1:124-5. http://dx.doi.org/10.1136/bmj.1.5219.124-a

2. Lowy FD. Staphylococcus aureus infections. N Engl J Med. 1998; 339:520-32. http://dx.doi.org/10.1056/NEJM199808203390806

3. Naimi TS, LeDell KH, Como-Sabetti K, Borchardt SM, Boxrud DJ, Etienne J, et al. Comparison of community- and health care-associated methicillin-resistant Staphylococcus aureus infection. JAMA. 2003;290:2976-84. http://dx.doi.org/10.1001/ jama.290.22.2976

4. McDougal LK, Steward CD, Killgore GE, Chaitram JM, McAllister SK, Tenover FC. Pulsed-field gel electrophoresis typing of oxacillin-resistant Staphylococcus aureus isolates from the United States: establishing a national database. J Clin Microbiol. 2003;41:5113-20. http://dx.doi.org/10.1128/ JCM.41.11.5113-5120.2003

5. Taylor AJ, Lappi V, Wolfgang WJ, Lapierre P, Palumbo MJ, Medus C, et al. Characterization of foodborne outbreaks of Salmonella enterica serovar Enteritidis with whole-genome sequencing single nucleotide polymorphism-based analysis for surveillance and outbreak detection. J Clin Microbiol. 2015;53:3334-40. http://dx.doi.org/10.1128/JCM.01280-15

6. Larsen MV, Cosentino S, Rasmussen S, Friis C, Hasman H, Marvig RL, et al. Multilocus sequence typing of total-genomesequenced bacteria. J Clin Microbiol. 2012;50:1355-61. http://dx.doi.org/10.1128/JCM.06094-11

7. Clinical and Laboratory Standards Institute. Performance standards for antimicrobial susceptibility testing. 26th ed. (M100S). Wayne (PA): The Institute; 2016.

8. Pereira LA, Harnett GB, Hodge MM, Cattell JA, Speers DJ. Real-time PCR assay for detection of blaZ genes in Staphylococcus aureus clinical isolates. J Clin Microbiol. 2014;52:1259-61. http://dx.doi.org/10.1128/JCM.03413-13

9. David MZ, Boyle-Vavra S, Zychowski DL, Daum RS. Methicillinsusceptible Staphylococcus aureus as a predominantly healthcareassociated pathogen: a possible reversal of roles? PLoS One. 2011;6:e18217. http://dx.doi.org/10.1371/journal.pone.0018217

10. Wang JT, Hsu LY, Lauderdale TL, Fan WC, Wang FD. Comparison of outcomes among adult patients with nosocomial bacteremia caused by methicillin-susceptible and methicillinresistant Staphylococcus aureus: a retrospective cohort study. PLoS One. 2015;10:e0144710. http://dx.doi.org/10.1371/ journal.pone. 0144710

11. Sicot N, Khanafer N, Meyssonnier V, Dumitrescu O, Tristan A, Bes M, et al. Methicillin resistance is not a predictor of severity in community-acquired Staphylococcus aureus necrotizing pneumoniaresults of a prospective observational study. Clin Microbiol Infect. 2013;19:E142-8. http://dx.doi.org/10.1111/1469-0691.12022

12. Hsiang MS, Shiau R, Nadle J, Chan L, Lee B, Chambers HF, et al. Epidemiologic similarities in pediatric community-associated methicillin-resistant and methicillin-sensitive Staphylococcus aureus in the San Francisco Bay Area. J Pediatric Infect Dis Soc. 2012;1:200-11. http://dx.doi.org/10.1093/jpids/pis061

13. Staphylococcus Laboratory, Statens Serum Institut. Annual report on Staphylococcus aureus bacteraemia cases in Denmark, 2012. Staphylococcus Laboratory, Statens Serum Institut, Copenhagen [cited 2018 Sep 28]. http://www.ssi.dk/ /media/Indhold/DK\%20 -\%20dansk/Smitteberedskab/Referencelaboratorier/ Stafylokoklaboratoriet/Rapport $\% 202012 \% 20 \% 20$ part $\% 20$ II.ashx.

14. Richter SS, Doern GV, Heilmann KP, Miner S, Tendolkar S, Riahi F, et al. Detection and prevalence of penicillin-susceptible Staphylococcus aureus in the United States in 2013. J Clin Microbiol. 2016;54:812-4. http://dx.doi.org/10.1128/ JCM.03109-15

15. Centers for Disease Control and Prevention. Healthcare-associated infections in the United States, 2006-2016: a story of progress, January 5, 2018 [cited 2018 Apr 25]. https://www.cdc.gov/hai/ surveillance/data-reports/data-summary-assessing-progress.html

Address for correspondence: Kathryn Como-Sabetti, Emerging Infections Unit, Minnesota Department of Health, 625 Robert St N, St. Paul, MN 55155, USA; email: kathy.como-sabetti@state.mn.us 